\title{
Sustainable Development Goal 3.6 and road traffic injury surveillance, Nigeria West Africa, 2013-2016
}

\section{Obafemi J. Babalola, Patrick Nguku, Merissa A. Yellman}

Africa Epidemiology Network, Nigeria

\section{Objective}

This study aimed to describe RTC characteristics and trends in Nigeria and determine progress towards halving RTC-related deaths/injuries by 2020 [i.e., Sustainable Development Goal (SDG) Target 3.6].

\section{Introduction}

Globally, road traffic crashes (RTCs) annually kill 1.3 million people and injure 20-50 million others. Nigeria accounts for an estimated $15 \%$ of RTC deaths in the WHO African Region.

\section{Methods}

We abstracted, cleaned, and analyzed RTC surveillance data routinely collected from crash scenes from 2013-2016. Federal Road Safety Corps (FRSC) is the lead agency for RTC surveillance and regularly collates data from the 6 geopolitical zones (which contain the 36 Nigerian States and the Federal Capital Territory). We defined road traffic injury as a fatal or non-fatal injury incurred from collision on a public road involving $\geq 1$ moving vehicle(s). We calculated descriptive statistics, frequencies, and proportions to describe RTC characteristics and trends.

\section{Results}

From 2013-2016, 283,949 persons were involved in 42,813 RTCs, resulting in 23,412 deaths and 127,264 injuries. Twenty-eight percent of RTCs involved $\geq 1$ fatality. Ninety percent of persons involved in RTCs were $\geq 18$ years old, with a male-female ratio of 3:1. The same proportion was also found for RTC deaths and for injuries. Despite containing only $15 \%$ of the country's population, the North-Central geopolitical zone accounted for 37\% of RTCs. The most common cause of RTCs was speed violations (26\%). From 2013-2016, there were reductions of 30\% for RTCs, $22 \%$ for number of fatalities, and $25 \%$ for number of injuries.

\section{Conclusions}

Nigeria reduced RTC deaths/injuries and achieved modest progress toward SDG Target 3.6. To further progress, FRSC can help by enhancing enforcement of speed violations and by educating road users about road safety practices. Also, they could investigate why certain geographical areas had disproportionate amounts of RTCs, deaths, and injuries. 